\title{
FRACTIONAL NEGATIVE BINOMIAL AND PÓLYA PROCESSES
}

BY

PALANIAPPAN VELLAISAMY (MUMbai) AND ADITYA MAHESHWARI* (INDORE)

\begin{abstract}
In this paper, we define a fractional negative binomial process (FNBP) by replacing the Poisson process by a fractional Poisson process (FPP) in the gamma subordinated form of the negative binomial process. It is shown that the one-dimensional distributions of the FPP and the FNBP are not infinitely divisible. Also, the space fractional Pólya process (SFPP) is defined by replacing the rate parameter $\lambda$ by a gamma random variable in the definition of the space fractional Poisson process. The properties of the FNBP and the SFPP and the connections to PDEs governing the density of the FNBP and the SFPP are also investigated.
\end{abstract}

2010 AMS Mathematics Subject Classification: Primary: 60G22; Secondary: 60G51.

Key words and phrases: Fractional negative binomial process, fractional Pólya process, fractional Poisson process, infinite divisibility, Lévy process, PDEs.

\section{INTRODUCTION}

The fractional generalizations of classical stochastic processes have received considerable attention by researchers in the recent years. These generalizations have found applications in several disciplines such as control theory, quantum physics, option pricing, actuarial science and reliability. For example, the fractional Poisson processes (FPPs) have been used recently in [15] to define a new family of quantum coherent states as well as the fractional generalization of Bell polynomials, Bell numbers and Stirling's numbers of the second kind. Also, a new renewal risk model, which is non-stationary and has the long-range dependence property, is defined using the FPP in [6]. In this paper, we define a fractional generalization of the negative binomial process and a space fractional version of the Pólya process. Quite recently, a fractional generalization of the negative binomial process has been defined in [3] and [4]. We introduce here a different generalization of the negative binomial process. It is known that the negative binomial process

* The research of AM was supported by UGC, Govt. of India grant F. 2-2/98 (SA-1). 
can be viewed as a Poisson process time-changed by a gamma subordinator. Let $\alpha>0, p>0$, and $\{\Gamma(t)\}_{t \geqslant 0}$ be a gamma process, where $\Gamma(t) \sim G(\alpha, p t)$, which denotes the gamma distribution with scale parameter $\alpha^{-1}$ and shape parameter $p t$. Let

$$
Q(t, \lambda)=N(\Gamma(t), \lambda), \quad t \geqslant 0,
$$

where $\{N(t, \lambda)\}_{t \geqslant 0}$ is a Poisson process with intensity $\lambda>0$. Then $\{Q(t, \lambda)\}_{t \geqslant 0}$ is called the negative binomial process, and $Q(t, \lambda) \sim \mathrm{NB}(p t, \eta)$ the negative binomial distribution with parameters $p t$ and $\eta=\lambda /(\alpha+\lambda)$ (see Section 2.2). For $0<\beta<1$, let $\left\{D_{\beta}(t)\right\}_{t \geqslant 0}$ be a $\beta$-stable subordinator, and $\left\{E_{\beta}(t)\right\}_{t \geqslant 0}$ be its (right-continuous) inverse stable subordinator defined by

$$
E_{\beta}(t)=\inf \left\{s>0: D_{\beta}(s)>t\right\}, \quad t>0 .
$$

A natural generalization of $\{Q(t, \lambda)\}_{t \geqslant 0}$ is to consider

$$
Q_{\beta}(t, \lambda)=N_{\beta}(\Gamma(t), \lambda), \quad t \geqslant 0,
$$

where $\left\{N_{\beta}(t, \lambda)\right\}_{t \geqslant 0}$ is the FPP (see [14], [20]). We call $\left\{Q_{\beta}(t, \lambda)\right\}_{t \geqslant 0}$ the fractional negative binomial process (FNBP). We will show that this process is different from the FNBP discussed in [3] and [4]. It is known that the Pólya process is obtained by replacing the parameter $\lambda$ by a gamma random variable in the definition of the Poisson process $\{N(t, \lambda)\}_{t \geqslant 0}$. Let $\Gamma \sim G(\alpha, p)$ and $W^{\Gamma}(t)=$ $N(t, \Gamma)$, where $\Gamma$ is independent of $\{N(t, \lambda)\}_{t \geqslant 0}$. Then $\left\{W^{\Gamma}(t)\right\}_{t \geqslant 0}$ is called the Pólya process. However, a fractional version of the Pólya process has not been addressed in the literature before. Recently, in [22], a space fractional Poisson process $\left\{\widetilde{N}_{\beta}(t, \lambda)\right\}_{t \geqslant 0}$, where $\widetilde{N}_{\beta}(t, \lambda)=N\left(D_{\beta}(t), \lambda\right)$, is introduced and its properties are investigated. We here introduce, as a fractional generalization of the Pólya process, the space fractional Pólya process (SFPP) defined by $\widetilde{W}_{\beta}^{\Gamma}(t)=\widetilde{N}_{\beta}(t, \Gamma)$ for $t \geqslant 0$.

The paper is organized as follows. In Section $\square$, some preliminary notation and results are stated. In Section 及, we discuss the infinite divisibility of the FPP $\left\{N_{\beta}(t, \lambda)\right\}_{t \geqslant 0}$ and also that of $\left\{N\left(E_{\beta}^{* n}(t), \lambda\right)\right\}_{t \geqslant 0}$, where $E_{\beta}^{* n}(t)$ is the $n$-iterated process of inverse stable subordinators. In Section 4 , we define the FNBP, compute its one-dimensional distributions and discuss their properties. It is shown that their one-dimensional distributions are not infinitely divisible, and they solve certain fractional PDEs. In Section [, we define the SFPP and show that it has stationary increments and is stochastically continuous. However, it does not have independent increments and hence is not a Lévy process. The fractional PDEs governed by the SFPP with respect to both the variables $t$ and $p$ are also discussed.

\section{PRELIMINARIES}

In this section, we introduce the notation and the results that will be used later. Let $\mathbb{Z}_{+}=\{0,1, \ldots\}$ be the set of nonnegative integers. 
2.1. Some special functions. We start with some special functions that will be required later.

(i) The Mittag-Leffler function $L_{\beta}(z)$ is defined as (see [8])

$$
L_{\beta}(z)=\sum_{k=0}^{\infty} \frac{z^{k}}{\Gamma(1+\beta k)}, \quad \beta, z \in \mathbb{C} \text { and } \operatorname{Re}(\beta)>0 .
$$

(ii) For $z \in \mathbb{C}$ and $0<\beta<1$, the $M$-Wright function $M_{\beta}(z)$ (see [10], [17]]) is defined as

$$
M_{\beta}(z)=\sum_{n=0}^{\infty} \frac{(-z)^{n}}{n ! \Gamma(-\beta n+(1-\beta))}=\frac{1}{\pi} \sum_{n=1}^{\infty} \frac{(-z)^{n-1}}{(n-1) !} \Gamma(\beta n) \sin (\pi \beta n) .
$$

Let $p, q \in \mathbb{Z}_{+}$. Also, for $0 \leqslant i \leqslant p, 0 \leqslant j \leqslant q$, let $a_{i}, b_{j}, z \in \mathbb{C}$.

(iii) Let $\alpha_{i}$ and $\beta_{j}$ be reals. The generalized Wright function is defined, under certain conditions (see [ए]], eq. (1.1)), as

$$
{ }_{p} \psi_{q}(z) \equiv{ }_{p} \psi_{q}\left[z \mid \begin{array}{c}
\left(a_{i}, \alpha_{i}\right)_{1, p} \\
\left(b_{j}, \beta_{j}\right)_{1, q}
\end{array}\right]=\sum_{k=0}^{\infty} \frac{\prod_{i=1}^{p} \Gamma\left(a_{i}+\alpha_{i} k\right)}{\prod_{j=1}^{q} \Gamma\left(b_{j}+\beta_{j} k\right)} \frac{z^{k}}{k !} .
$$

(iv) Let $A_{i}$ and $B_{j}$ be positive reals. The $H$-function (see [19], Section 1.2) is defined in terms of the Mellin-Barnes type integral as

$$
H_{p, q}^{m, n}(z) \equiv H_{p, q}^{m, n}\left[z \mid \begin{array}{c}
\left(a_{i}, A_{i}\right)_{1, p} \\
\left(b_{j}, B_{j}\right)_{1, q}
\end{array}\right]=\frac{1}{2 \pi i} \int_{L} \chi_{p, q}^{m, n}(s) z^{-s} d s
$$

where $z \neq 0$ and $z^{-s}=\exp [-s\{\ln |z|+i \arg (z)\}]$. Here, $\ln |z|$ represents the natural logarithm of $|z|$ and $\arg (z)$ is not necessarily the principal value. Also, an empty product is interpreted as unity and

$$
\chi_{p, q}^{m, n}(s)=\frac{\prod_{i=1}^{m} \Gamma\left(1-a_{i}-A_{i} s\right) \prod_{j=1}^{n} \Gamma\left(b_{j}+B_{j} s\right)}{\prod_{i=m+1}^{p} \Gamma\left(a_{i}+A_{i} s\right) \prod_{j=n+1}^{q} \Gamma\left(1-b_{j}+B_{j} s\right)},
$$

where $m, n, p$ and $q$ are nonnegative integers such that $0 \leqslant m \leqslant p, 1 \leqslant n \leqslant q$ and

$$
A_{i}\left(b_{j}+l\right) \neq B_{j}\left(a_{i}-k-1\right)
$$

for $l, k \in \mathbb{Z}_{+}, 1 \leqslant i \leqslant m$ and $1 \leqslant j \leqslant n$. The contour $L$ in (2.3) runs from $c-i \infty$ to $c+i \infty$ and separates the poles $s_{j, l}=-\left(\left(b_{j}+l\right) / B_{j}\right)$ of $\Gamma\left(b_{j}+B_{j} s\right)$ from 
the poles $w_{i, k}=\left(\left(1-a_{i}+k\right) / A_{i}\right)$ of $\Gamma\left(1-a_{i}-A_{i} s\right)$, where $1 \leqslant i \leqslant m$ and $1 \leqslant j \leqslant n$.

It is known that, under certain conditions (see [II], eq. (5.2)), the generalized Wright function ${ }_{p} \psi_{q}$ given in (2.2) satisfies

$$
{ }_{p} \psi_{q}\left[z \mid \begin{array}{l}
\left(a_{i}, A_{i}\right)_{(1, p)} \\
\left(b_{j}, B_{j}\right)_{(1, q)}
\end{array}\right]=H_{p, q+1}^{1, p}\left[\begin{array}{l|c}
\left(1-a_{i}, A_{i}\right)_{(1, p)} \\
(0,1),\left(1-b_{j}, B_{j}\right)_{(1, q)}
\end{array}\right] .
$$

2.2. Some elementary distributions. Let $\{N(t, \lambda)\}_{t \geqslant 0}$ be a Poisson process with rate $\lambda>0$, so that

$$
p(n \mid t, \lambda)=\mathbb{P}[N(t, \lambda)=n]=\frac{(\lambda t)^{n} e^{-\lambda t}}{n !}, \quad n \in \mathbb{Z}_{+} .
$$

For $\alpha>0, p>0$, let $\{\Gamma(t)\}_{t \geqslant 0}$ be a gamma process, where $\Gamma(t) \sim G(\alpha, p t)$ with density

$$
g(y \mid \alpha, p t)=\frac{\alpha^{p t}}{\Gamma(p t)} y^{p t-1} e^{-\alpha y}, \quad y>0 .
$$

We say that a random variable $X$ follows a negative binomial distribution with parameters $\alpha>0$ and $0<\eta<1$, denoted by $\mathrm{NB}(\alpha, \eta)$, if

$$
\mathbb{P}[X=n]=\left(\begin{array}{c}
n+\alpha-1 \\
n
\end{array}\right) \eta^{n}(1-\eta)^{\alpha}, \quad n \in \mathbb{Z}_{+}
$$

If $\alpha$ is a natural number, then $X$ denotes the number of successes before the $\alpha$-th failure in a sequence of Bernoulli trials with success probability $\eta$.

We say that $X$ follows a logarithmic series distribution with parameter $\eta$, denoted by $L S(\eta)$, if

$$
\mathbb{P}[X=n]=\frac{-\eta^{n}}{n \ln (1-\eta)}, \quad n \in \mathbb{Z}_{+} \backslash\{0\} .
$$

Let $\left\{D_{\beta}(t)\right\}_{t \geqslant 0}$ be a $\beta$-stable subordinator. Then the density of $D_{\beta}(t)$ is (see [10], eq. (4.7))

$$
g_{\beta}(x, t)=\beta t x^{-(\beta+1)} M_{\beta}\left(t x^{-\beta}\right), \quad x>0 .
$$

Let $\left\{E_{\beta}(t)\right\}_{t \geqslant 0}$ be the inverse $\beta$-stable subordinator defined in (IL.J). Then the density of $E_{\beta}(t)$ is (see [110], eq. (5.7))

$$
h_{\beta}(x, t)=t^{-\beta} M_{\beta}\left(t^{-\beta} x\right), \quad x>0 .
$$


2.3. Some fractional derivatives. Let $A C[a, b]$ be the space of functions $f$ which are absolutely continuous on $[a, b]$ and

$$
A C^{n}[a, b]=\left\{f:[a, b] \rightarrow \mathbb{R} ; \frac{d^{n-1}}{d t^{n-1}} f(t) \in A C[a, b]\right\},
$$

where $A C^{1}[a, b]=A C[a, b]$.

Definition 2.1. Let $m \in \mathbb{Z}_{+} \backslash\{0\}$ and $\nu \geqslant 0$. If $f(t) \in A C^{m}[0, T]$, then the (left-hand) Riemann-Liouville fractional derivative $\partial_{t}^{\nu} f$ of $f$ (see [12], Lemma 2.2) is defined by (with $\partial_{t}^{0} f=f$ )

$$
\partial_{t}^{\nu} f(t):= \begin{cases}\frac{1}{\Gamma(m-\nu)} \frac{d^{m}}{d t^{m}} \int_{0}^{t} \frac{f(s)}{(t-s)^{\nu-m+1}} d s, & m-1<\nu<m, \\ \frac{d^{m}}{d t^{m}} f(t), & \nu=m .\end{cases}
$$

Definition 2.2. Let $m \in \mathbb{Z}_{+} \backslash\{0\}$ and $\nu \geqslant 0$. If $f(t) \in A C^{m}[0, T]$, then the (left-hand) Caputo fractional derivative $D_{t}^{\nu} f$ of $f$ (see [12], Theorem 2.1) is defined by (with $D_{t}^{0} f=f$ )

$$
D_{t}^{\nu} f(t):= \begin{cases}\frac{1}{\Gamma(m-\nu)} \int_{0}^{t} \frac{f^{(m)}(s)}{(t-s)^{\nu-m+1}} d s, & m-1<\nu<m, \\ \frac{d^{m}}{d t^{m}} f(t), & \nu=m .\end{cases}
$$

The relation between the Riemann-Liouville fractional derivative and the Caputo fractional derivative is (see [12], eq. (2.4.6))

$$
\partial_{t}^{\nu} f(t)=D_{t}^{\nu} f(t)+\sum_{k=0}^{m-1} \frac{t^{k-\nu}}{\Gamma(k-\nu+1)} f^{(k)}\left(0^{+}\right),
$$

where $f^{(k)}\left(0^{+}\right):=\lim _{t \rightarrow 0^{+}} \frac{d^{k}}{d t^{k}} f(t)$.

\section{FRACTIONAL POISSON PROCESS}

Let $0<\beta \leqslant 1$. The fractional Poisson process (FPP) $\left\{N_{\beta}(t, \lambda)\right\}_{t \geqslant 0}$, which is a generalization of the Poisson process $\{N(t, \lambda)\}_{t \geqslant 0}$, is defined to be a stochastic process for which $p_{\beta}(n \mid t, \lambda)=\mathbb{P}\left[N_{\beta}(t, \lambda)=n\right]$ satisfies (see [14], [18], [20])

$$
\begin{aligned}
& D_{t}^{\beta} p_{\beta}(n \mid t, \lambda)=-\lambda\left[p_{\beta}(n \mid t, \lambda)-p_{\beta}(n-1 \mid t, \lambda)\right] \quad \text { for } n \geqslant 1, \\
& D_{t}^{\beta} p_{\beta}(0 \mid t, \lambda)=-\lambda p_{\beta}(0 \mid t, \lambda)
\end{aligned}
$$


with $p_{\beta}(n \mid 0, \lambda)=1$ if $n=0$ and $p_{\beta}(n \mid 0, \lambda)=0$ if $n \geqslant 1$. Here, $D_{t}^{\beta}$ denotes the Caputo fractional derivative defined in ([2.8). The pmf $p_{\beta}(n \mid t, \lambda)$ for the FPP is given by (see [14], [20])

$$
p_{\beta}(n \mid t, \lambda)=\frac{\left(\lambda t^{\beta}\right)^{n}}{n !} \sum_{k=0}^{\infty} \frac{(n+k) !}{k !} \frac{\left(-\lambda t^{\beta}\right)^{k}}{\Gamma(\beta(k+n)+1)} .
$$

Note that the equation (3.]) can also be represented as

$$
p_{\beta}(n \mid t, \lambda)=\frac{\left(\lambda t^{\beta}\right)^{n}}{n !}{ }_{1} \psi_{1}\left[-\lambda t^{\beta} \mid \begin{array}{c}
(n+1,1) \\
(n \beta+1, \beta)
\end{array}\right],
$$

by using the generalized Wright function defined in (2.2) .

The mean and the variance of the FPP are given by (see [14])

$$
\begin{aligned}
\mathbb{E}\left[N_{\beta}(t, \lambda)\right] & =q t^{\beta}, \\
\operatorname{Var}\left[N_{\beta}(t, \lambda)\right] & =q t^{\beta}\left[1+q t^{\beta}\left(\frac{\beta B(\beta, 1 / 2)}{2^{2 \beta-1}}-1\right)\right],
\end{aligned}
$$

where $q=\lambda / \Gamma(1+\beta)$ and $B(a, b)$ denotes the beta function. An alternative form for $\operatorname{Var}\left[N_{\beta}(t, \lambda)\right]$ is given in [5], eq. (2.8), as

$$
\operatorname{Var}\left[N_{\beta}(t, \lambda)\right]=q t^{\beta}+\frac{\left(\lambda t^{\beta}\right)^{2}}{\beta}\left(\frac{1}{\Gamma(2 \beta)}-\frac{1}{\beta \Gamma^{2}(\beta)}\right) .
$$

Note that (B.4) follows from (B.3) using the Legendre's duplication formula (see [1], p. 22)

$$
\Gamma(2 a) \Gamma(1 / 2)=2^{2 a-1} \Gamma(a) \Gamma(a+1 / 2), \quad a>0 .
$$

It is also known that (see [20]) when $0<\beta<1$,

$$
N_{\beta}(t, \lambda) \stackrel{d}{=} N\left(E_{\beta}(t), \lambda\right),
$$

where $\left\{E_{\beta}(t)\right\}_{t \geqslant 0}$ is the inverse $\beta$-stable subordinator and is independent of $\{N(t, \lambda)\}_{t \geqslant 0}$. First we establish an important property of the FPP.

THEOREM 3.1. Let $0<\beta<1$. The one-dimensional distributions of the FPP $\left\{N_{\beta}(t, \lambda)\right\}_{t \geqslant 0}$ are not infinitely divisible (i.d.).

P r o o f. Since the sample paths of $\left\{D_{\beta}(t)\right\}_{t \geqslant 0}$ are strictly increasing, the process $\left\{E_{\beta}(t)\right\}_{t \geqslant 0}$ has continuous sample paths. Further,

$$
\mathbb{P}\left[E_{\beta}(t) \leqslant x\right]=\mathbb{P}\left[D_{\beta}(x) \geqslant t\right] .
$$


It is well known that a $\beta$-stable process is self-similar with index $1 / \beta$, that is,

$$
D_{\beta}(c t) \stackrel{d}{=} c^{1 / \beta} D_{\beta}(t), \quad c>0 .
$$

Hence,

$$
\begin{aligned}
\mathbb{P}\left[E_{\beta}(c t) \leqslant x\right]=\mathbb{P}\left[D_{\beta}(x) \geqslant c t\right] & =\mathbb{P}\left[\frac{1}{c} D_{\beta}(x) \geqslant t\right]=\mathbb{P}\left[D_{\beta}\left(x / c^{\beta}\right) \geqslant t\right] \\
& =\mathbb{P}\left[E_{\beta}(t) \leqslant x / c^{\beta}\right]=\mathbb{P}\left[c^{\beta} E_{\beta}(t) \leqslant x\right] .
\end{aligned}
$$

That is,

$$
E_{\beta}(c t) \stackrel{d}{=} c^{\beta} E_{\beta}(t),
$$

showing that $E_{\beta}(t)$ is also self-similar with index $\beta$.

Observe now that

$$
N_{\beta}(t, \lambda) \stackrel{d}{=} N\left(E_{\beta}(t), \lambda\right) \stackrel{d}{=} N\left(t^{\beta} E_{\beta}(1), \lambda\right) .
$$

By the renewal theorem for the Poisson process,

$$
\lim _{t \rightarrow \infty} \frac{N(t, \lambda)}{t}=\lambda \text { a.s. }
$$

This implies, since $E_{\beta}(t)$ is independent of $\{N(t, \lambda)\}_{t \geqslant 0}$,

$$
\lim _{t \rightarrow \infty} \frac{N\left(t^{\beta} E_{\beta}(1), \lambda\right)}{t^{\beta}}=E_{\beta}(1) \lim _{t \rightarrow \infty} \frac{N\left(t^{\beta} E_{\beta}(1), \lambda\right)}{t^{\beta} E_{\beta}(1)}=\lambda E_{\beta}(1) \text { a.s. }
$$

since $E_{\beta}(1)>0$ a.s. Hence, for $0<\beta<1$,

$$
\frac{N_{\beta}(t, \lambda)}{t^{\beta}} \stackrel{\mathcal{L}}{\longrightarrow} \lambda E_{\beta}(1)
$$

where $\stackrel{\mathcal{L}}{\rightarrow}$ denotes convergence in law. Assume now that $N_{\beta}(t, \lambda)$ is i.d. Then $N_{\beta}(t, \lambda) / t^{\beta}$ is also i.d. for each $t$. Since the limit of a sequence of i.d. random variables is also i.d. (see [23], Lemma 7.8), it follows that $\lambda E_{\beta}(1)$ or, equivalently, $E_{\beta}(1)$ is i.d., which is a contradiction since $E_{\beta}(t)$ is not i.d. for $t>0$ (see [25]). Hence, the result follows.

Let $\left\{E_{\beta_{1}}(t)\right\}, \ldots,\left\{E_{\beta_{n}}(t)\right\}$ be independent inverse stable subordinators and $\beta=\beta_{1} \beta_{2} \ldots \beta_{n}$. Consider the $n$-iterated process $\left\{E_{\beta}^{* n}(t)\right\}$, where $E_{\beta}^{* n}(t)=$ $E_{\beta_{1}} \circ E_{\beta_{2}} \circ \ldots \circ E_{\beta_{n}}(t)$ and, for example, $E_{\beta_{1}} \circ E_{\beta_{2}}(t)=E_{\beta_{1}}\left(E_{\beta_{2}}(t)\right)$. By [25], Remark 2.5, we infer that $E_{\beta}^{* n}(t)$ is not i.d. We have the following result for the Poisson process with time change $E_{\beta}^{* n}(t)$. 
THEOREM 3.2. The one-dimensional distributions of the subordinated Poisson process $\left\{N\left(E_{\beta}^{* n}(t), \lambda\right)\right\}_{t \geqslant 0}$ are not i.d.

Pro of. For some $c>0$ and using (B.6), we have

$$
E_{\beta_{1}}\left(E_{\beta_{2}}(c t)\right) \stackrel{d}{=} E_{\beta_{1}}\left(c^{\beta_{2}} E_{\beta_{2}}(t)\right) \stackrel{d}{=} c^{\beta_{1} \beta_{2}} E_{\beta_{1}}\left(E_{\beta_{2}}(t)\right) .
$$

Thus, in general, for $\beta=\beta_{1} \beta_{2} \ldots \beta_{n}$, we have $E_{\beta}^{* n}(c t) \stackrel{d}{=} c^{\beta} E_{\beta}^{* n}(t)$ and hence

$$
\frac{N\left(E_{\beta}^{* n}(t), \lambda\right)}{t^{\beta}} \stackrel{\mathcal{L}}{\longrightarrow} \lambda E_{\beta}^{* n}(1)
$$

which is not i.d., and hence the result follows.

\section{FRACTIONAL NEGATIVE BINOMIAL PROCESS}

4.1. Definition and properties. Let $\{N(t, \lambda)\}_{t \geqslant 0}$ be a Poisson process and $\{\Gamma(t)\}_{t \geqslant 0}$ be a gamma subordinator, where $\Gamma(t) \sim G(\alpha, p t)$ as defined in (2.4), and be independent of $\{N(t, \lambda)\}_{t \geqslant 0}$. The negative binomial process $\{Q(t, \lambda)\}_{t \geqslant 0}$ $=\{N(\Gamma(t), \lambda)\}_{t \geqslant 0}$ is a subordinated Poisson process (see [9], [13]) with

$$
\begin{aligned}
& \mathbb{P}[Q(t, \lambda)=n]=\delta(n \mid \alpha, p t, \lambda)=\frac{\alpha^{p t} \lambda^{n}}{n ! \Gamma(p t)} \int_{0}^{\infty} y^{n+p t-1} e^{-y(\alpha+\lambda)} d y \\
& \quad=\left(\begin{array}{c}
n+p t-1 \\
n
\end{array}\right)\left(\frac{\alpha}{\alpha+\lambda}\right)^{p t}\left(\frac{\lambda}{\alpha+\lambda}\right)^{n}=\left(\begin{array}{c}
n+p t-1 \\
n
\end{array}\right) \eta^{n}(1-\eta)^{p t},
\end{aligned}
$$

where $\eta=\lambda /(\alpha+\lambda)$. That is, $Q(t, \lambda) \sim \mathrm{NB}(p t, \eta)$ for $t>0$, defined in ([2.5)).

DEFINITION 4.1. The fractional negative binomial process (FNBP) is defined as

$$
Q_{\beta}(t, \lambda)=N_{\beta}(\Gamma(t), \lambda), \quad t \geqslant 0
$$

where $\left\{N_{\beta}(t, \lambda)\right\}_{t \geqslant 0}$ is an FPP and is independent of $\{\Gamma(t)\}_{t \geqslant 0}$.

Let $g(y \mid \alpha, p t)$ denote the pdf of $\Gamma(t)$, given in (2.4). Then,

$$
\mathbb{P}\left[Q_{\beta}(t, \lambda)=n\right]=\delta_{\beta}(n \mid \alpha, p t, \lambda)=\int_{0}^{\infty} p_{\beta}(n \mid y, \lambda) g(y \mid \alpha, p t) d y
$$




$$
\begin{aligned}
& =\frac{\lambda^{n}}{n !} \sum_{k=0}^{\infty}(-\lambda)^{k} \frac{(n+k) !}{k !} \frac{1}{\Gamma(\beta(n+k)+1)} \frac{\alpha^{p t}}{\Gamma(p t)} \int_{0}^{\infty} e^{-\alpha y} y^{(n+k) \beta+p t-1} d y \\
& =\frac{\lambda^{n}}{n !} \sum_{k=0}^{\infty}(-\lambda)^{k} \frac{(n+k) !}{k !} \frac{1}{\Gamma(\beta(n+k)+1)} \frac{\alpha^{p t}}{\Gamma(p t)} \frac{\Gamma((n+k) \beta+p t)}{\alpha^{(n+k) \beta+p t}} \\
& =\left(\frac{\lambda}{\alpha^{\beta}}\right)^{n} \frac{1}{n !} \sum_{k=0}^{\infty} \frac{(n+k) !}{k !} \frac{\Gamma((n+k) \beta+p t)}{\Gamma(p t) \Gamma(\beta(n+k)+1)}\left(\frac{-\lambda}{\alpha^{\beta}}\right)^{k} \\
& =\frac{1}{\Gamma(p t) n !}\left(\frac{\lambda}{\alpha^{\beta}}\right)^{n}{ }_{2} \psi_{1}\left[\begin{array}{cc}
-\lambda \\
\alpha^{\beta}
\end{array} \begin{array}{c}
(n+1,1), \\
(n \beta+1, \beta)
\end{array}\right.
\end{aligned}
$$

Assume $\left|-\lambda / \alpha^{\beta}\right|<1$. Then, by Theorem 1(b) in [W] and with $\delta=1^{-1} \beta^{-\beta} \beta^{\beta}=1$, $\Delta=\beta-\beta-1=-1$, the associated series of ${ }_{2} \psi_{1}$ function in the last equality in (4.1) converges. Thus, we have proved the following result.

THEOREM 4.1. Let $0<\beta \leqslant 1,0<\lambda<\alpha^{\beta}$, where $\alpha>0$. Then the FNBP $\left\{Q_{\beta}(t, \lambda)\right\}_{t \geqslant 0}$ has the one-dimensional distributions

$$
\delta_{\beta}(n \mid \alpha, p t, \lambda)=\frac{1}{\Gamma(p t) n !}\left(\frac{\lambda}{\alpha^{\beta}}\right)^{n}{ }_{2} \psi_{1}\left[\frac{-\lambda}{\alpha^{\beta}} \mid \begin{array}{ll}
(n+1,1), & (n \beta+p t, \beta) \\
(n \beta+1, \beta) &
\end{array}\right]
$$

and, consequently,

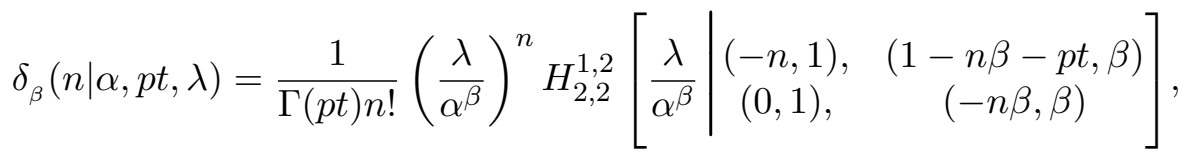

where $n \in \mathbb{Z}_{+}$, and $H_{2,2}^{1,2}$ is the $H$-function defined in (‥3).

When $\beta=1$, we can see that $\delta_{1}(n \mid \alpha, p t, \lambda)$ reduces to the $\operatorname{pmf}$ of $\operatorname{NB}(p t, \eta)$ distribution. We next show that $\delta_{\beta}(n \mid \alpha, p t, \lambda)$ is indeed a pmf for $0<\beta<1$ also. Note that

$$
\begin{aligned}
\sum_{n=0}^{\infty} \delta_{\beta}(n \mid \alpha, p t, \lambda) & =\sum_{n=0}^{\infty}\left(\frac{\lambda}{\alpha^{\beta}}\right)^{n} \sum_{k=0}^{\infty}\left(\begin{array}{c}
n+k \\
k
\end{array}\right) \frac{\Gamma((n+k) \beta+p t)}{\Gamma(p t) \Gamma((n+k) \beta+1)}\left(\frac{-\lambda}{\alpha^{\beta}}\right)^{k} \\
& =\sum_{n=0}^{\infty} \frac{\left(\lambda / \alpha^{\beta}\right)^{n}}{n ! \Gamma(p t)} \sum_{k=n}^{\infty} \frac{k !}{(k-n) !} \frac{\Gamma(k \beta+p t)}{\Gamma(k \beta+1)}\left(\frac{-\lambda}{\alpha^{\beta}}\right)^{k-n} \\
& =\frac{1}{\Gamma(p t)} \sum_{k=0}^{\infty} \frac{\Gamma(k \beta+p t)}{\Gamma(k \beta+1)} \sum_{n=0}^{k}\left(\begin{array}{c}
k \\
n
\end{array}\right)\left(\frac{\lambda}{\alpha^{\beta}}\right)^{n}\left(\frac{-\lambda}{\alpha^{\beta}}\right)^{k-n}=1
\end{aligned}
$$

since only the term corresponding to $k=0$ remains. 
REMARK 4.1. Let $0<\alpha^{\beta}<\lambda$. Then, using the representation given in (B.5) and from (2.6), we also obtain

$$
\begin{aligned}
\delta_{\beta}(n \mid \alpha, p t, \lambda) & =\int_{0}^{\infty} \int_{0}^{\infty} p(n \mid x, \lambda) h_{\beta}(x, y) g(y \mid \alpha, p t) d x d y \\
& =\frac{\alpha^{\beta}}{\lambda \Gamma(p t)} \sum_{k=0}^{\infty}\left(\begin{array}{c}
n+k \\
n
\end{array}\right) \frac{\Gamma(p t-\beta-\beta k)}{\Gamma(-\beta k+(1-\beta))}\left(-\frac{\alpha^{\beta}}{\lambda}\right)^{k} \\
& =\frac{\alpha^{\beta}}{n ! \lambda \Gamma(p t)^{2}} \psi_{1}\left[\begin{array}{c|c}
-\alpha^{\beta} \\
\lambda
\end{array} \begin{array}{c}
(n+1,1), \quad(p t-\beta,-\beta) \\
(1-\beta,-\beta)
\end{array}\right] .
\end{aligned}
$$

Henceforth, let us put

$$
q=\lambda / \Gamma(1+\beta), \quad d_{1}=2 \lambda^{2} / \Gamma(2 \beta+1) \quad \text { and } \quad d_{2}=\beta q^{2} B(\beta, 1+\beta) .
$$

THEOREM 4.2. The mean, variance and autocovariance functions of the $F N B P\left\{Q_{\beta}(t, \lambda)\right\}_{t \geqslant 0}$ are given by

(i) $\mathbb{E}\left[Q_{\beta}(t, \lambda)\right]=q \frac{\Gamma(p t+\beta)}{\alpha^{\beta} \Gamma(p t)}=q \mathbb{E}\left[\Gamma^{\beta}(t)\right] \sim q\left(\frac{p t}{\alpha}\right)^{\beta}=\left(\frac{p}{\alpha}\right)^{\beta} \mathbb{E}\left[N_{\beta}(t, \lambda)\right]$, for large $t$,

(ii) $\operatorname{Var}\left[Q_{\beta}(t, \lambda)\right]=\frac{q \Gamma(p t+\beta)}{\alpha^{\beta} \Gamma(p t)}\left(1-\frac{q \Gamma(p t+\beta)}{\alpha^{\beta} \Gamma(p t)}\right)+\frac{d_{1} \Gamma(p t+2 \beta)}{\alpha^{2 \beta} \Gamma(p t)}$,

(iii) $\operatorname{Cov}\left[Q_{\beta}(s, \lambda), Q_{\beta}(t, \lambda)\right]=q \frac{\Gamma(p s+\beta)}{\alpha^{\beta} \Gamma(p s)}+d_{2} \frac{\Gamma(p s+2 \beta)}{\alpha^{2 \beta} \Gamma(p s)}$

$$
-q^{2} \frac{\Gamma(p s+\beta)}{\alpha^{2 \beta} \Gamma(p s)} \frac{\Gamma(p t+\beta)}{\Gamma(p t)}+q^{2} \beta \mathbb{E}\left[\Gamma^{2 \beta}(t) B(\beta, 1+\beta ; \Gamma(s) / \Gamma(t))\right] .
$$

Proof. For simplicity, the parameter $\lambda$ is suppressed in $\left\{N_{\beta}(t, \lambda)\right\}_{t \geqslant 0}$ and $\left\{Q_{\beta}(t, \lambda)\right\}_{t \geqslant 0}$, when no confusion arises. First note that

$$
\mathbb{E}\left[\Gamma^{l}(t)\right]=\frac{1}{\alpha^{l}} \frac{\Gamma(p t+l)}{\Gamma(p t)}, \quad l>0 .
$$

Using a conditioning argument and the equation (3.2), we get

$$
\mathbb{E}\left[Q_{\beta}(t)\right]=\frac{q}{\alpha^{\beta}} \frac{\Gamma(p t+\beta)}{\Gamma(p t)}=q \mathbb{E}\left[\Gamma^{\beta}(t)\right] .
$$

By Stirling's formula, $(\Gamma(p t+\beta) / \Gamma(p t)) \sim(p t)^{\beta}$ for large $t$, and so we get

$$
\mathbb{E}\left[N_{\beta}(\Gamma(t))\right] \sim(p / \alpha)^{\beta} q t^{\beta}=(p / \alpha)^{\beta} \mathbb{E}\left[N_{\beta}(t, \lambda)\right],
$$

which proves part (i). 
Using (B.2) and (B.4), we get

$$
\begin{aligned}
\operatorname{Var}\left[Q_{\beta}(t)\right] & =\operatorname{Var}\left[\mathbb{E}\left[N_{\beta}(\Gamma(t)) \mid \Gamma(t)\right]\right]+\mathbb{E}\left[\operatorname{Var}\left[N_{\beta}(\Gamma(t)) \mid \Gamma(t)\right]\right] \\
& =q \mathbb{E}\left[\Gamma^{\beta}(t)\right]\left(1-q \mathbb{E}\left[\Gamma^{\beta}(t)\right]\right)+d_{1} \mathbb{E}\left[\Gamma^{2 \beta}(t)\right] \\
& =\frac{q \Gamma(p t+\beta)}{\alpha^{\beta} \Gamma(p t)}\left(1-\frac{q \Gamma(p t+\beta)}{\alpha^{\beta} \Gamma(p t)}\right)+\frac{d_{1} \Gamma(p t+2 \beta)}{\alpha^{2 \beta} \Gamma(p t)},
\end{aligned}
$$

using (4.4). This proves part (ii).

Next, from [16], eq. (14), we get, for $0<s \leqslant t$,

$$
\operatorname{Cov}\left[N_{\beta}(s), N_{\beta}(t)\right]=q s^{\beta}+d_{2} s^{2 \beta}+q^{2}\left[\beta t^{2 \beta} B(\beta, 1+\beta ; s / t)-(s t)^{\beta}\right],
$$

where $B(a, b ; x)=\int_{0}^{x} t^{a-1}(1-t)^{b-1} d t, 0<x<1$, is the incomplete beta function. Hence, from (B.2),

$$
\mathbb{E}\left[N_{\beta}(s) N_{\beta}(t)\right]=q s^{\beta}+d_{2} s^{2 \beta}+q^{2} \beta\left[t^{2 \beta} B(\beta, 1+\beta ; s / t)\right],
$$

which leads to

$$
\begin{aligned}
& \mathbb{E}\left[Q_{\beta}(s) Q_{\beta}(t)\right]=\mathbb{E}\left[\mathbb{E}\left[N_{\beta}(\Gamma(s)) N_{\beta}(\Gamma(t)) \mid \Gamma(s), \Gamma(t)\right]\right] \\
& \quad=q \mathbb{E}\left[\Gamma^{\beta}(s)\right]+d_{2} \mathbb{E}\left[\Gamma^{2 \beta}(s)\right]+\beta q^{2} \mathbb{E}\left[\Gamma^{2 \beta}(t) B(\beta, 1+\beta ; \Gamma(s) / \Gamma(t))\right] .
\end{aligned}
$$

Hence, using (4.5), we get

$$
\begin{aligned}
\operatorname{Cov}\left[Q_{\beta}(s), Q_{\beta}(t)\right]= & q \mathbb{E}\left[\Gamma^{\beta}(s)\right]+d_{2} \mathbb{E}\left[\Gamma^{2 \beta}(s)\right]-q^{2} \mathbb{E}\left[\Gamma^{\beta}(s)\right] \mathbb{E}\left[\Gamma^{\beta}(t)\right] \\
& +\beta q^{2} \mathbb{E}\left[\Gamma^{2 \beta}(t) B(\beta, 1+\beta ; \Gamma(s) / \Gamma(t))\right] .
\end{aligned}
$$

Using (4.4), we obtain part (iii).

REMARK 4.2. A stochastic process $\{X(t)\}_{t \geqslant 0}$ is said be overdispersed if $\operatorname{Var}[X(t)]-\mathbb{E}[X(t)]>0$ for all $t \geqslant 0$ (see [4] $)$. Now, for the FNBP,

$$
\begin{aligned}
\operatorname{Var}\left[Q_{\beta}(t)\right]- & \mathbb{E}\left[Q_{\beta}(t)\right]=\frac{d_{1} \Gamma(p t+2 \beta)}{\alpha^{2 \beta} \Gamma(p t)}-\left(\frac{q \Gamma(p t+\beta)}{\alpha^{\beta} \Gamma(p t)}\right)^{2} \\
& =\frac{\lambda^{2}}{\beta}\left(\frac{\mathbb{E}\left[\Gamma^{2 \beta}(t)\right]}{\Gamma(2 \beta)}-\frac{\left(\mathbb{E}\left[\Gamma^{\beta}(t)\right]\right)^{2}}{\beta \Gamma^{2}(\beta)}\right) \\
& \left.\geqslant\left(\lambda \mathbb{E}\left[\Gamma^{\beta}(t)\right]\right)^{2} Z(\beta) \quad \text { (because } \mathbb{E}\left[\Gamma^{2 \beta}(t)\right] \geqslant\left(\mathbb{E}\left[\Gamma^{\beta}(t)\right]\right)^{2}\right),
\end{aligned}
$$

where $Z(\beta)=\beta^{-1}\left(1 / \Gamma(2 \beta)-1 / \beta \Gamma^{2}(\beta)\right)>0$ for all $\beta \in(0,1)$ (see [4], Section 3.1). Hence, the FNBP exhibits overdispersion. 
The next result shows that the FNBP is not i.d.

THEOREM 4.3. The one-dimensional distributions of the FNBP $\left\{Q_{\beta}(t, \lambda)\right\}_{t \geqslant 0}$ are not i.d.

Pro of. Since $E_{\beta}(t) \stackrel{d}{=} t^{\beta} E_{\beta}(1)$, we have

$$
Q_{\beta}(t, \lambda)=N\left(E_{\beta}(\Gamma(t)), \lambda\right) \stackrel{d}{=} N\left(\Gamma^{\beta}(t) E_{\beta}(1), \lambda\right) .
$$

Using (B.7), we obtain

$$
\begin{aligned}
\lim _{t \rightarrow \infty} \frac{N\left(\Gamma^{\beta}(t) E_{\beta}(1), \lambda\right)}{t^{\beta}} & =\lim _{t \rightarrow \infty} \frac{N\left(\Gamma^{\beta}(t) E_{\beta}(1), \lambda\right)}{\Gamma^{\beta}(t)}\left(\frac{\Gamma(t)}{t}\right)^{\beta} \\
& =\lambda E_{\beta}(1)(\mathbb{E}[\Gamma(1)])^{\beta}=\lambda E_{\beta}(1)\left(\frac{p}{\alpha}\right)^{\beta} \text { a.s. }
\end{aligned}
$$

since $\Gamma(t) \rightarrow \infty$ and $\Gamma(t) / t \rightarrow \mathbb{E}[\Gamma(1)]$ a.s., as $t \rightarrow \infty$. The result follows by contradiction since $E_{\beta}(1)$ is not i.d.

REMARK 4.3. (i) In fact, the above result can be generalized for any subordinator $T(t)$ with $\mathbb{E}[T(1)]<\infty$. For a subordinator, the $S L L N$ for Lévy processes yields $\lim _{t \rightarrow \infty} T(t) / t=\mathbb{E}[T(1)]$ a.s. Thus,

$$
\frac{N_{\beta}(T(t), \lambda)}{t^{\beta}} \stackrel{\mathcal{L}}{\longrightarrow} \lambda E_{\beta}(1)(\mathbb{E}[T(1)])^{\beta}
$$

which is not i.d.

(ii) Since

$$
\frac{N\left(E_{\beta}^{* n}(\Gamma(t)), \lambda\right)}{t^{\beta_{1} \beta_{2} \cdots \beta_{n}}} \stackrel{\mathcal{L}}{\longrightarrow} \lambda E_{\beta}^{* n}(1)(\mathbb{E}[\Gamma(1)])^{\beta_{1} \beta_{2} \cdots \beta_{n}} \quad \text { as } t \rightarrow \infty,
$$

it follows that the distributions of $\left\{N\left(E_{\beta}^{* n}(\Gamma(t)), \lambda\right)\right\}_{t \geqslant 0}$ are also not i.d.

REMARK 4.4. Let $q(x, t)$ be the pdf of $E_{\beta}(\Gamma(t))$, and $h_{\beta}(x, t)$ be the pdf of $E_{\beta}(t)$. It is known (see [21], [7]) that $\mathbb{E}\left[e^{-s E_{\beta}(t)}\right]=L_{\beta}\left[-s t^{\beta}\right]$, where $L_{\beta}(z)$ is the Mittag-Leffler function defined in (‥J). Then the LT of the process $\left\{E_{\beta}(\Gamma(t))\right\}_{t \geqslant 0}$ can be computed as

$$
\begin{aligned}
\mathbb{E}\left[e^{-s E_{\beta}(\Gamma(t))}\right]=\int_{0}^{\infty} e^{-s x} q(x, t) d x=\int_{0}^{\infty} \int_{0}^{\infty} e^{-s x} h_{\beta}(x, y) g(y \mid \alpha, p t) d y d x \\
=\int_{0}^{\infty} L_{\beta}\left(-s y^{\beta}\right) g(y \mid \alpha, p t) d y=\sum_{k=0}^{\infty} \frac{\Gamma(\beta k+p t)}{\Gamma(p t) \Gamma(1+\beta k)}\left(\frac{-s}{\alpha^{\beta}}\right)^{k} \\
=\frac{1}{\Gamma(p t)^{2}} \psi_{1}\left[\begin{array}{ll}
\frac{-s}{\alpha^{\beta}} \mid \begin{array}{cc}
(p t, \beta), \\
(1, \beta)
\end{array} & (1,1)
\end{array}\right] .
\end{aligned}
$$


Using (4.6), we obtain the LT of $\left\{Q_{\beta}(t, \lambda)\right\}_{t \geqslant 0}$ as

$$
\begin{aligned}
\mathbb{E}\left[e^{-s Q_{\beta}(t, \lambda)}\right] & =\mathbb{E}\left[\mathbb{E}\left[\exp \left(-\lambda E_{\beta}(\Gamma(t))\left(1-e^{-s}\right)\right) \mid E_{\beta}(\Gamma(t))\right]\right] \\
& =\frac{1}{\Gamma(p t)^{2}} \psi_{1}\left[\frac{\lambda\left(e^{-s}-1\right)}{\alpha^{\beta}} \mid \begin{array}{c}
(p t, \beta), \quad(1,1) \\
(1, \beta)
\end{array}\right] .
\end{aligned}
$$

Recently, Beghin [3] and Beghin and Macci [4] also studied the FNBP. For $0<\beta<1$ and $0<\eta<1$, they define the FNBP as

$$
X_{1}(t)=\sum_{i=1}^{N_{\beta}(t,-\ln (1-\eta))} Y_{i} \quad \text { and } \quad X_{2}(t)=N\left(\Gamma_{\beta}^{*}(t), 1\right)
$$

in [4] and [3], respectively, where the $Y_{i}$ 's are $L S(\eta)$-distributed random variables independent of $\left\{N_{\beta}(t, \lambda)\right\}_{t \geqslant 0},\left\{\Gamma_{\beta}^{*}(t)\right\}_{t \geqslant 0}=\left\{\Gamma^{*}\left(E_{\beta}(t)\right)\right\}_{t \geqslant 0}$ is the fractional gamma process (see [3]) and $\Gamma^{*}(t) \sim G(\alpha, t)$. It is assumed here that $\left\{\Gamma^{*}(t)\right\}_{t \geqslant 0}$ and $\left\{E_{\beta}(t)\right\}_{t \geqslant 0}$ are independent processes. Note that when $\beta=1$,

$$
X_{1}(t) \sim \mathrm{NB}(t, \eta) \quad \text { and } \quad X_{2}(t) \sim \mathrm{NB}(t, 1 /(1+\alpha)) .
$$

Observe that our definition of the FNBP is

$$
Q_{\beta}(t, \lambda):=N_{\beta}(\Gamma(t), \lambda) \stackrel{d}{=} N\left(E_{\beta}(\Gamma(t)), \lambda\right) .
$$

When $\beta=1, Q_{1}(t, \lambda)=N(\Gamma(t), \lambda) \sim \mathrm{NB}(p t, \lambda /(\lambda+\alpha)), t>0$ (see, e.g., [26]). Let, for $i \geqslant 1, Y_{i}^{*} \stackrel{\text { iid }}{\sim} L S(\lambda /(\alpha+\lambda))$. Then it can be seen that

$$
Q_{1}(t, \lambda) \stackrel{d}{=} \sum_{i=1}^{N(p t,-\ln (\alpha /(\lambda+\alpha)))} Y_{i}^{*},
$$

where $\left\{Y_{i}^{*}\right\}_{i \geqslant 1}$ and $\{N(t, \lambda)\}_{t \geqslant 0}$ are independent. It can be seen that our process is different from theirs.

LeMma 4.1. Let $0<\beta<1$. Then the processes $\left\{N\left(\Gamma\left(E_{\beta}(t)\right), \lambda\right)\right\}_{t \geqslant 0}$ and $\left\{Q_{\beta}(t, \lambda)\right\}_{t \geqslant 0}$ are different.

Proof. It is sufficient to prove that the one-dimensional distributions of the processes $\left\{\Gamma\left(E_{\beta}(t)\right)\right\}_{t \geqslant 0}$ and $\left\{E_{\beta}(\Gamma(t))\right\}_{t \geqslant 0}$ are different. We compare the mean functions of the processes $\left\{\Gamma\left(E_{\beta}(t)\right)\right\}_{t \geqslant 0}$ and $\left\{E_{\beta}(\Gamma(t))\right\}_{t \geqslant 0}$. Using $\mathbb{E}\left[E_{\beta}(t)\right]=$ $t^{\beta} / \Gamma(1+\beta)$, we get

$$
\mathbb{E}\left[\Gamma\left(E_{\beta}(t)\right)\right]=\frac{p t^{\beta}}{\alpha \Gamma(1+\beta)}, \quad \mathbb{E}\left[E_{\beta}(\Gamma(t))\right]=\frac{\Gamma(p t+\beta)}{\alpha^{\beta} \Gamma(1+\beta) \Gamma(p t)},
$$

which are clearly different. This proves the result. 
4.2. Connections to PDEs. In this section, we discuss PDEs governed by the one-dimensional distributions of the FNBP.

THEOREM 4.4. Let $r \in \mathbb{Z}_{+} \backslash\{0\}$. The pmf (4.2) of the FNBP solves the following PDE:

$$
\frac{\partial^{r}}{\partial \lambda^{r}} \delta_{\beta}(n \mid \alpha, p t, \lambda)=
$$

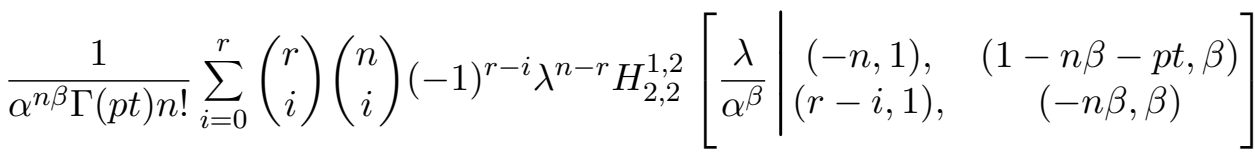

with

(4.8) $\delta_{\beta}(n \mid \alpha, 0, \lambda)=\left\{\begin{array}{ll}1, & n=0, \\ 0, & n \geqslant 1,\end{array} \quad\right.$ and $\quad \delta_{\beta}(n \mid \alpha, p t, \lambda)=0$ for all $n<0$.

Pr o o f. Note first that the $H$-function defined in (2.3) satisfies (see [19], Section (1.4.1)), for $r \in \mathbb{Z}_{+} \backslash\{0\}$,

$$
\begin{aligned}
& \frac{\partial^{r}}{\partial z^{r}}\left\{z^{-\left(\gamma \beta_{1} / B_{1}\right)} H_{p, q}^{m, n}\left[z^{\gamma} \mid \begin{array}{l}
\left(\alpha_{i}, A_{i}\right)_{(1, p)} \\
\left(\beta_{j}, B_{j}\right)_{(1, q)}
\end{array}\right]\right\} \\
& =\left(\frac{-\gamma}{B_{1}}\right)^{r} z^{-r-\left(\gamma \beta_{1} / B_{1}\right)} H_{p, q}^{m, n}\left[z^{\gamma} \mid \begin{array}{c}
\left(\alpha_{i}, A_{i}\right)_{(1, p)} \\
\left(r+\beta_{1}, B_{1}\right),\left(\beta_{j}, B_{j}\right)_{(2, q)}
\end{array}\right] .
\end{aligned}
$$

Taking $p=2, q=2, m=1, n=2, \alpha_{1}=-n, \alpha_{2}=1-n \beta-p t, \beta_{1}=0, \beta_{2}=$ $-n \beta, A_{1}=B_{1}=1, A_{2}=B_{2}=\beta$ and $\gamma=1$, we get

$$
\begin{aligned}
& \frac{\partial^{r}}{\partial z^{r}} H_{2,2}^{1,2}\left[z \mid \begin{array}{cc}
(-n, 1), & (1-n \beta-p t, \beta) \\
(0,1), & (-n \beta, \beta)
\end{array}\right] \\
&=(-1)^{r} z^{-r} H_{2,2}^{1,2}\left[z\left[\begin{array}{cc}
(-n, 1), & (1-n \beta-p t, \beta) \\
(r, 1), & (-n \beta, \beta)
\end{array}\right]\right.
\end{aligned}
$$

Now, differentiate $r$ times the right-hand side of (4.3) with respect to $\lambda$, use (4.9) and the Leibniz rule

$$
\frac{d^{r}}{d x^{r}}[u(x) v(x)]=\sum_{i=0}^{r}\left(\begin{array}{l}
r \\
i
\end{array}\right) \frac{d^{i}}{d x^{i}}(u(x)) \frac{d^{r-i}}{d x^{r-i}}(v(x))
$$

to obtain the result in (4.7). 
REMARK 4.5. When $r=1$, we get

$$
\begin{aligned}
& \frac{\partial}{\partial \lambda} \delta_{\beta}(n \mid \alpha, p t, \lambda) \\
= & \frac{n}{\lambda} \delta_{\beta}(n \mid \alpha, p t, \lambda)-\frac{1}{\lambda \Gamma(p t) n !}\left(\frac{\lambda}{\alpha^{\beta}}\right)^{n} H_{2,2}^{1,2}\left[\frac{\lambda}{\alpha^{\beta}} \begin{array}{cc}
(-n, 1), & (1-n \beta-p t, \beta) \\
(1,1), & (-n \beta, \beta)
\end{array}\right]
\end{aligned}
$$

with the initial condition given in (4.8).

Next, we obtain the fractional PDE in the time variable $t$ solved by the FNBP distributions.

LEMMA 4.2. The density of the gamma process $\Gamma(t) \sim G(\alpha, p t)$, given in (2.4), satisfies the following fractional differential equation for any $\nu \geqslant 0$ :

$$
\begin{aligned}
\partial_{t}^{\nu} g(y \mid \alpha, p t) & =p \partial_{t}^{\nu-1}(\log (\alpha y)-\psi(p t)) g(y \mid \alpha, p t), \quad y>0, \\
g(y \mid \alpha, 0) & =0
\end{aligned}
$$

where $\psi(x):=\Gamma^{\prime}(x) / \Gamma(x)$ is the digamma function and $\partial_{t}^{\nu}$ denotes the RiemannLiouville derivative defined in (2.7).

Pr o of. Note first that (see [24], eq. (3.6))

$$
\frac{1}{\Gamma(t)}=\frac{1}{2 \pi i} \int_{\mathbf{C}} e^{z} z^{-t} d z
$$

where $\mathbf{C}$ is the Hankel contour given in Figure $\mathbb{W}$.

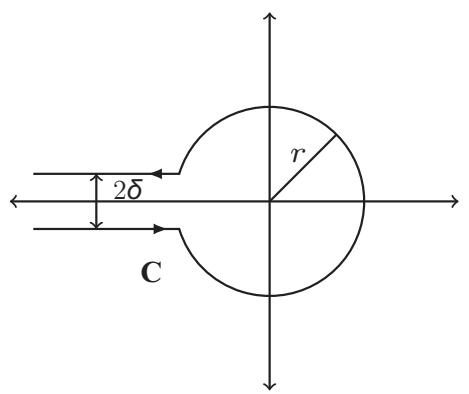

FIGURE 1. Hankel contour.

Let $\Gamma(a, x)=\int_{x}^{\infty} e^{-t} t^{a-1} d t, a>0, x>0$, denote the incomplete gamma function. Then, for $m-1<\nu<m, m \in \mathbb{Z}_{+} \backslash\{0\}$,

$\int_{0}^{t} \frac{(\alpha y / z)^{p s}}{(t-s)^{\nu+1-m}} d s=\left(\frac{\alpha y}{z}\right)^{p t}\left(p \log \frac{\alpha y}{z}\right)^{\nu-m}\left\{\Gamma(m-\nu)-\Gamma\left(m-\nu, p t \log \frac{\alpha y}{z}\right)\right\}$ 
and

$$
\frac{d}{d t} \Gamma\left(m-\nu, p t \log \frac{\alpha y}{z}\right)=-p\left(\frac{\alpha y}{z}\right)^{-p t}\left(p \log \frac{\alpha y}{z}\right)^{m-1-\nu} \log \left(\frac{\alpha y}{z}\right),
$$

which can be checked using Mathematica 8.0. Now, by definition, for $m-1<$ $\nu<m$,

$$
\begin{aligned}
& \partial_{t}^{\nu} g(y \mid \alpha, p t) \\
& =\frac{1}{\Gamma(m-\nu)} \frac{d^{m}}{d t^{m}} \int_{0}^{t} \frac{\alpha^{p s} y^{p s-1} e^{-\alpha y}}{\Gamma(p s)(t-s)^{\nu+1-m}} d s \\
& =\frac{\left(y e^{\alpha y}\right)^{-1}}{\Gamma(m-\nu)} \frac{d^{m}}{d t^{m}} \int_{0}^{t} \frac{(\alpha y)^{p s}}{(t-s)^{\nu+1-m}}\left(\frac{1}{2 \pi i} \int_{\mathbf{C}} e^{z} z^{-p s} d z\right) d s \quad \text { (from (4.TI)) } \\
& =\frac{\left(y e^{\alpha y}\right)^{-1}}{\Gamma(m-\nu)} \frac{d^{m}}{d t^{m}} \frac{1}{2 \pi i} \int_{\mathbf{C}} e^{z}\left(\int_{0}^{t} \frac{(\alpha y / z)^{p s}}{(t-s)^{\nu+1-m}} d s\right) d z \quad \text { (interchanging the order) } \\
& =\frac{\left(y e^{\alpha y}\right)^{-1}}{\Gamma(m-\nu)} \frac{d^{m}}{d t^{m}} \frac{1}{2 \pi i} \int_{\mathbf{C}} e^{z}\left(\frac{\alpha y}{z}\right)^{p t}\left(p \log \frac{\alpha y}{z}\right)^{\nu-m} \\
& \times\left\{\Gamma(m-\nu)-\Gamma\left(m-\nu, p t \log \frac{\alpha y}{z}\right)\right\} d z \quad(\text { from (4.12) }) \\
& =p \frac{\left(y e^{\alpha y}\right)^{-1}}{\Gamma(m-\nu)} \frac{d^{m-1}}{d t^{m-1}} \frac{1}{2 \pi i} \int_{\mathbf{C}} e^{z}\left(\frac{\alpha y}{z}\right)^{p t} \log \frac{\alpha y}{z}\left(p \log \frac{\alpha y}{z}\right)^{\nu-m} \\
& \times\left\{\Gamma(m-\nu)-\Gamma\left(m-\nu, p t \log \frac{\alpha y}{z}\right)\right\} d z \\
& -\frac{\left(y e^{\alpha y}\right)^{-1}}{\Gamma(m-\nu)} \frac{d^{m-1}}{d t^{m-1}} \frac{1}{2 \pi i} \int_{\mathbf{C}} e^{z}\left(\frac{\alpha y}{z}\right)^{p t}\left(p \log \frac{\alpha y}{z}\right)^{\nu-m} \\
& \times\left\{-p\left(\frac{\alpha y}{z}\right)^{-p t}\left(p \log \frac{\alpha y}{z}\right)^{m-1-\nu} \log \left(\frac{\alpha y}{z}\right)\right\} d z \quad \text { (from (4.13)) } \\
& =p \frac{\left(y e^{\alpha y}\right)^{-1}}{\Gamma(m-\nu)} \frac{d^{m-1}}{d t^{m-1}} \frac{1}{2 \pi i} \int_{\mathbf{C}} e^{z}\left(\frac{\alpha y}{z}\right)^{p t}(\log (\alpha y)-\log (z))\left(p \log \frac{\alpha y}{z}\right)^{\nu-m} \\
& \times\left\{\Gamma(m-\nu)-\Gamma\left(m-\nu, p t \log \frac{\alpha y}{z}\right)\right\} d z+\frac{\left(y e^{\alpha y}\right)^{-1}}{\Gamma(m-\nu)} \frac{d^{m-1}}{d t^{m-1}} \frac{1}{2 \pi i} \int_{\mathbf{C}} e^{z} d z \\
& =p \log (\alpha y) \frac{\left(y e^{\alpha y}\right)^{-1}}{\Gamma(m-\nu)} \frac{d^{m-1}}{d t^{m-1}} \frac{1}{2 \pi i} \int_{\mathbf{C}} e^{z}\left(\frac{\alpha y}{z}\right)^{p t}\left(p \log \frac{\alpha y}{z}\right)^{\nu-m} \\
& \times\left\{\Gamma(m-\nu)-\Gamma\left(m-\nu, p t \log \frac{\alpha y}{z}\right)\right\} d z \\
& -p \frac{\left(y e^{\alpha y}\right)^{-1}}{\Gamma(m-\nu)} \frac{d^{m-1}}{d t^{m-1}} \frac{1}{2 \pi i} \int_{\mathbf{C}} e^{z}\left(\frac{\alpha y}{z}\right)^{p t} \log (z)\left(p \log \frac{\alpha y}{z}\right)^{\nu-m} \\
& \left.\times\left\{\Gamma(m-\nu)-\Gamma\left(m-\nu, p t \log \frac{\alpha y}{z}\right)\right\} d z \quad \text { (because } \int_{\mathbf{C}} e^{z} d z=0\right)
\end{aligned}
$$


$=p \log (\alpha y) \partial_{t}^{\nu-1} g(y \mid \alpha, p t)-\frac{\left(y e^{\alpha y}\right)^{-1}}{\Gamma(m-\nu)} \frac{d^{m-1}}{d t^{m-1}} \frac{1}{2 \pi i} \int_{0}^{t} \frac{\alpha^{p s} y^{p s}}{(t-s)^{\nu+1-m}} \frac{d}{d s} \frac{1}{\Gamma(p s)} d s$

$=p \log (\alpha y) \partial_{t}^{\nu-1} g(y \mid \alpha, p t)-p \partial_{t}^{\nu-1}\{g(y \mid \alpha, p t) \psi(p t)\}$,

which proves the result.

The following corollary corresponds to the case $\nu=1$.

COROllary 4.1. The density (2.4) of the gamma process $\Gamma(t) \sim G(\alpha, p t)$ solves the following PDE in the time variable $t$ (with $g(y \mid \alpha, 0)=0)$ :

$$
\frac{\partial}{\partial t} g(y \mid \alpha, p t)=p(\log (\alpha y)-\psi(p t)) g(y \mid \alpha, p t), \quad y>0, t>0 .
$$

THEOREM 4.5. The pmf (4.2) of the FNBP solves the following fractional $P D E$ in the time variable $t$ :

$$
\begin{aligned}
\frac{1}{p} \partial_{t}^{\nu} \delta_{\beta}(n \mid \alpha, p t, \lambda)= & \partial_{t}^{\nu-1}(\log (\alpha)-\psi(p t)) \delta_{\beta}(n \mid \alpha, p t, \lambda) \\
& +\int_{0}^{\infty} p_{\beta}(n \mid y, \lambda) \log (y) \partial_{t}^{\nu-1} g(y \mid \alpha, p t) d y, \quad t>0,
\end{aligned}
$$

where $\delta_{\beta}(n \mid \alpha, 0, \lambda)=1$ if $n=0$ and is zero otherwise, and $p_{\beta}(n \mid t, \lambda)$ denotes the pmf of the FPP defined in (B.J).

Proof. Let $m-1<\nu<m$, where $m$ is a positive integer. Then

$$
\begin{aligned}
\partial_{t}^{\nu} \delta_{\beta}(n \mid \alpha, p t, \lambda) & =\partial_{t}^{\nu} \int_{0}^{\infty} p_{\beta}(n \mid y, \lambda) g(y \mid \alpha, p t) d y \\
& =\frac{1}{\Gamma(m-\nu)} \frac{d^{m}}{d t^{m}} \int_{0}^{t} \int_{0}^{\infty} p_{\beta}(n \mid y, \lambda) \frac{g(y \mid \alpha, p s)}{(t-s)^{\nu+1-m}} d y d s
\end{aligned}
$$

and, consequently,

$$
\begin{aligned}
\partial_{t}^{\nu} \delta_{\beta}(n \mid \alpha, p t, \lambda) & =\frac{1}{\Gamma(m-\nu)} \int_{0}^{\infty} p_{\beta}(n \mid y, \lambda) \frac{d^{m}}{d t^{m}} \int_{0}^{t} \frac{g(y \mid \alpha, p s)}{(t-s)^{\nu+1-m}} d s d y \\
& =\int_{0}^{\infty} p_{\beta}(n \mid y, \lambda) \partial_{t}^{\nu} g(y \mid \alpha, p t) d y
\end{aligned}
$$

The change in the order of integration in (4.14) can be justified, by using the Fubini-Tonelli theorem, as follows:

$$
\left|\int_{0}^{t} \int_{0}^{\infty} p_{\beta}(n \mid y, \lambda) \frac{g(y \mid \alpha, p s)}{(t-s)^{\nu+1-m}} d y d s\right|
$$




$$
\begin{aligned}
& \leqslant \int_{0}^{t} \frac{1}{(t-s)^{\nu+1-m}} \int_{0}^{\infty}\left|p_{\beta}(n \mid y, \lambda) g(y \mid \alpha, p s)\right| d y d s \\
& \leqslant \int_{0}^{t} \frac{1}{(t-s)^{\nu+1-m}} d s=\frac{t^{(m-\nu)}}{m-\nu}<\infty .
\end{aligned}
$$

The result now follows from (4.10) and the second line of (4.14).

\section{SPACE FRACTIONAL PÓLYA PROCESS}

5.1. Pólya process. Recall that $\{N(t, \lambda)\}_{t \geqslant 0}$ represents a Poisson process with rate $\lambda>0$ and $\mathbb{P}[N(t, \lambda)=n]=p(n \mid t, \lambda)$. The Pólya process $\left\{W^{\Gamma}(t)\right\}_{t \geqslant 0}:=$ $\{N(t, \Gamma)\}_{t \geqslant 0}$ is obtained by replacing $\lambda$ by a random variable $\Gamma$, with density $g(x \mid \alpha, p)$ given in (2.4), which is independent of $\{N(t, \lambda)\}_{t \geqslant 0}$. Then the pmf $\eta(n \mid t, \alpha, p)=\mathbb{P}\left[W^{\Gamma}(t)=n\right]$ of the Pólya process is given by

$$
\eta(n \mid t, \alpha, p)=\int_{0}^{\infty} p(n \mid t, x) g(x \mid \alpha, p) d x=\frac{t^{n}}{n !} \frac{\Gamma(n+p)}{\Gamma(p)} \frac{\alpha^{p}}{(t+\alpha)^{p+n}}
$$

which is the pmf of $\operatorname{NB}\left(p, \frac{t}{\alpha+t}\right)$. Since the pmf $p(n \mid t, \lambda)$ of the Poisson process satisfies

$$
\frac{\partial}{\partial t} p(n \mid t, \lambda)=-\lambda[p(n \mid t, \lambda)-p(n-1 \mid t, \lambda)]
$$

we have

$$
\frac{\partial}{\partial t} \eta(n \mid t, \alpha, p)=\int_{0}^{\infty}-x[p(n \mid t, x)-p(n-1 \mid t, x)] g(x \mid \alpha, p) d x .
$$

Now,

$$
\int_{0}^{\infty} x p(n \mid t, x) g(x \mid \alpha, p) d x=\frac{t^{n} \alpha^{p}}{n ! \Gamma(p)} \frac{\Gamma(n+p+1)}{(t+\alpha)^{n+p+1}}=\frac{n+p}{t+\alpha} \eta(n \mid t, \alpha, p), \quad n \in \mathbb{Z}_{+},
$$

which follows by using (5.1). Putting (5.3) in (5.2), we obtain

$$
\frac{\partial}{\partial t} \eta(n \mid t, \alpha, p)=-\frac{n+p}{t+\alpha} \eta(n \mid t, \alpha, p)+\frac{n-1+p}{t+\alpha} \eta(n-1 \mid t, \alpha, p), \quad n \geqslant 0
$$

with $\eta(n \mid t, \alpha, p)=0$ for $n<0$, which is the underlying difference-differential equation satisfied by the Pólya process.

Observe that the negative binomial process $\{Q(t, \lambda)\}_{t \geqslant 0}$ is a Lévy process (see [13], [9]) so that it has independent increments. However, the Pólya process $\left\{W^{\Gamma}(t)\right\}_{t \geqslant 0}$ is not a Lévy process, as it does not have independent increments (see Remark 5.2 below). 
5.2. Space fractional Pólya process. The space fractional Poisson process $\left\{\widetilde{N}_{\beta}(t, \lambda)\right\}_{t \geqslant 0}$, which is a generalization of the Poisson process $\{N(t, \lambda)\}_{t \geqslant 0}$, defined in [22], can be viewed as (see [22], Remark 2.3)

$$
\tilde{N}_{\beta}(t, \lambda) \stackrel{d}{=} N\left(D_{\beta}(t), \lambda\right)
$$

where $D_{\beta}(t)$ is a $\beta$-stable subordinator with $0<\beta<1$. The pmf of the space fractional Poisson process is given by (see [22], Theorem 2.2)

$$
\widetilde{p}_{\beta}(n \mid t, \lambda)=\mathbb{P}\left[\widetilde{N}_{\beta}(t, \lambda)=n\right]=\frac{(-1)^{n}}{n !} \sum_{k=0}^{\infty} \frac{\left(-\lambda^{\beta} t\right)^{k}}{k !} \frac{\Gamma(\beta k+1)}{\Gamma(\beta k+1-n)}, \quad t \geqslant 0 .
$$

It solves the fractional difference-differential equation ([22], eq. (2.4)) defined by

$$
\begin{aligned}
\frac{\partial}{\partial t} \widetilde{p}_{\beta}(n \mid t, \lambda) & =-\lambda^{\beta}\left(1-B_{n}\right)^{\beta} \widetilde{p}_{\beta}(n \mid t, \lambda), \quad \beta \in(0,1], \\
\widetilde{p}_{\beta}(n \mid 0, \lambda) & = \begin{cases}1 & \text { for } n=0, \\
0 & \text { for } n>0,\end{cases}
\end{aligned}
$$

where $B_{x}$ is the backward shift operator defined by $B_{x} u(x, t)=u(x-1, t)$.

Definition 5.1. Assume $\left\{D_{\beta}(t)\right\}_{t \geqslant 0}$ is a $\beta$-stable subordinator, and $\Gamma$ is a $G(\alpha, p)$-distributed random variable, independent of $\left\{D_{\beta}(t)\right\}_{t \geqslant 0}$ and $\{N(t, \lambda)\}_{t \geqslant 0}$. Then the space fractional Pólya process (SFPP) $\left\{\widetilde{W}_{\beta}^{\Gamma}(t)\right\}_{t \geqslant 0}$ is defined as

$$
\widetilde{W}_{\beta}^{\Gamma}(t):=\widetilde{N}_{\beta}(t, \Gamma)=N\left(D_{\beta}(t), \Gamma\right), \quad t \geqslant 0 .
$$

THEOREM 5.1. Let $0<\beta \leqslant 1$. The one-dimensional distributions of the SFPP are, for $n \in \mathbb{Z}_{+}$,

$$
\widetilde{\eta}_{\beta}(n \mid t, \alpha, p)=\mathbb{P}\left[\widetilde{W}_{\beta}^{\Gamma}(t)=n\right]=\frac{1}{\Gamma(p)} \frac{(-1)^{n}}{n !}{ }_{2} \psi_{1}\left[\begin{array}{c|c}
t & \begin{array}{c}
(1, \beta), \\
\alpha^{\beta}
\end{array}(p, \beta)
\end{array}\right],
$$

where ${ }_{2} \psi_{1}$ denotes the generalized Wright function defined in (2.2).

Proof. Observe that

$$
\begin{aligned}
\widetilde{\eta}_{\beta}(n \mid t, \alpha, p) & =\mathbb{E}\left[\mathbb{P}\left[\widetilde{W}_{\beta}^{\Gamma}(t)=n \mid \Gamma\right]\right]=\int_{0}^{\infty} \widetilde{p}_{\beta}(n \mid t, y) g(y \mid \alpha, p) d y \\
& =\int_{0}^{\infty} \frac{(-1)^{n}}{n !} \sum_{k=0}^{\infty} \frac{\left(-y^{\beta} t\right)^{k}}{k !} \frac{\Gamma(\beta k+1)}{\Gamma(\beta k+1-n)} \frac{\alpha^{p}}{\Gamma(p)} y^{p-1} e^{-\alpha y} d y \\
& =\frac{(-1)^{n}}{n !} \sum_{k=0}^{\infty} \frac{(-t)^{k}}{k !} \frac{\Gamma(\beta k+1)}{\Gamma(\beta k+1-n)} \frac{\alpha^{p}}{\Gamma(p)} \int_{0}^{\infty} y^{\beta k+p-1} e^{-\alpha y} d y
\end{aligned}
$$




$$
\begin{aligned}
& =\frac{1}{\Gamma(p)} \frac{(-1)^{n}}{n !} \sum_{k=0}^{\infty} \frac{\Gamma(\beta k+1) \Gamma(\beta k+p)}{\Gamma(\beta k+1-n)} \frac{\left(-t / \alpha^{\beta}\right)^{k}}{k !} \\
& =\frac{1}{\Gamma(p)} \frac{(-1)^{n}}{n !}{ }_{2} \psi_{1}\left[-\frac{t}{\alpha^{\beta}} \mid \begin{array}{cc}
(1, \beta), & (1-n, \beta)
\end{array}\right] \text {. }
\end{aligned}
$$

When $\beta=1$, we get

$$
\begin{aligned}
\widetilde{\eta}_{1}(n \mid t, \alpha, p) & =\frac{1}{\Gamma(p)} \frac{(-1)^{n}}{n !} \sum_{k=0}^{\infty} \frac{\Gamma(k+1) \Gamma(k+p)}{\Gamma(k+1-n)} \frac{(-t / \alpha)^{k}}{k !} \\
& =\frac{1}{\Gamma(p)} \frac{(-1)^{n}}{n !} \sum_{k=0}^{\infty} \frac{\Gamma(k+p)}{(k-n) !}\left(\frac{-t}{\alpha}\right)^{k} \quad(\text { put } j=k-n) \\
& =\frac{1}{\Gamma(p)} \frac{(-1)^{n}}{n !} \sum_{n+j=0}^{\infty} \frac{\Gamma(n+j+p)}{j !}\left(\frac{-t}{\alpha}\right)^{n+j} \\
& =\frac{\Gamma(n+p)}{\Gamma(p) \alpha^{n}} \frac{t^{n}}{n !} \sum_{j=0}^{\infty} \frac{\Gamma(n+j+p)}{\Gamma(j+1) \Gamma(n+p)}\left(\frac{-t}{\alpha}\right)^{j} \\
& =\frac{t^{n}}{n !} \frac{\Gamma(n+p)}{\Gamma(p)} \frac{\alpha^{p}}{(t+\alpha)^{p+n}},
\end{aligned}
$$

which is the pmf of $\mathrm{NB}\left(p, \frac{t}{\alpha+t}\right)$, as expected.

REMARK 5.1. Let us consider the time fractional generalization of the Pólya process, namely $\left\{N_{\beta}(t, \Gamma)\right\}_{t \geqslant 0}$, where $\Gamma$ is independent of $\left\{N_{\beta}(t, \lambda)\right\}_{t \geqslant 0}$, which would also be of interest. It seems difficult to compute its pmf given by

$$
\mathbb{P}\left[N_{\beta}(t, \Gamma)=n\right]=\int_{0}^{\infty} p_{\beta}(n \mid t, x) g(x \mid \alpha, p) d x .
$$

THEOREM 5.2. The SFPP $\left\{\widetilde{W}_{\beta}^{\Gamma}(t)\right\}_{t \geqslant 0}$ has stationary increments and is stochastically continuous.

Pr o o f. Consider first the Pólya process $\left\{W^{\Gamma}(t)\right\}_{t \geqslant 0}$.

(i) Stationary increments. Let $B$ be a Borel set. Then, for $t \geqslant 0$ and $s>0$,

$$
\begin{aligned}
\mathbb{P}\left[W^{\Gamma}(t+s)-W^{\Gamma}(s) \in B\right] & =\mathbb{E}[\mathbb{P}[N(t+s, \Gamma)-N(s, \Gamma) \in B \mid \Gamma]] \\
& =\mathbb{E}[\mathbb{P}[N(t, \Gamma) \in B \mid \Gamma]]=\mathbb{P}\left[W^{\Gamma}(t) \in B\right],
\end{aligned}
$$

showing that $\left\{W^{\Gamma}(t)\right\}_{t \geqslant 0}$ has stationary increments. It is known that the time change of a process with stationary increments by a process with stationary in- 
crements has stationary increments (see [2], Theorem 1.3.25). Since $\left\{D_{\beta}(t)\right\}_{t \geqslant 0}$ is a Lévy process, $\left\{\widetilde{W}_{\beta}^{\Gamma}(t)\right\}_{t \geqslant 0}$ also has stationary increments.

(ii) Stochastic continuity. Note first that for any process $\{X(t)\}_{t \geqslant 0}$ with stationary increments,

$$
\lim _{t \rightarrow s} \mathbb{P}[|X(t)-X(s)|>a]=0 \Rightarrow \lim _{t \rightarrow 0} \mathbb{P}[|X(t)|>a]=0 \quad \text { for } a>0 .
$$

Suppose that $W^{\Gamma}(t)=N(t, \Gamma)$ is not stochastically continuous. Then there exist $a, \epsilon>0$ and a sequence $\left(t_{n}\right)_{n \geqslant 1}$ with $t_{n} \rightarrow 0$ such that $\mathbb{P}\left[W^{\Gamma}\left(t_{n}\right)>a\right] \geqslant \epsilon$ for $n \geqslant 1$. Now choose $\lambda_{0}$ large enough such that $\int_{\lambda_{0}}^{\infty} g(\lambda \mid \alpha, p) d \lambda<\epsilon / 2$, and since the Poisson process $\{N(t, \lambda)\}_{t \geqslant 0}$ is stochastically continuous, there exists, for given $\lambda_{0}$ and $a>0$, some $\delta>0$ such that $\mathbb{P}\left[N\left(t, \lambda_{0}\right)>a\right]<\epsilon / 2$ for all $t \in(0, \delta)$. Thus, for $t \in(0, \delta)$,

$$
\begin{aligned}
\mathbb{P}\left[W^{\Gamma}(t)>a\right] & =\mathbb{E}[\mathbb{P}[N(t, \Gamma)>a \mid \Gamma]]=\int_{0}^{\infty} \mathbb{P}[N(t, \lambda)>a] g(\lambda \mid \alpha, p) d \lambda \\
& =\int_{0}^{\lambda_{0}} \mathbb{P}[N(t, \lambda)>a] g(\lambda \mid \alpha, p) d \lambda+\int_{\lambda_{0}}^{\infty} \mathbb{P}[N(t, \lambda)>a] g(\lambda \mid \alpha, p) d \lambda .
\end{aligned}
$$

Since $\mathbb{P}[N(t, \lambda)>a]$ is an increasing function of $\lambda$, we have

$$
\mathbb{P}[N(t, \lambda)>a] \leqslant \mathbb{P}\left[N\left(t, \lambda_{0}\right)>a\right]<\epsilon / 2 \quad \text { for } 0<\lambda \leqslant \lambda_{0} .
$$

Hence, for $t \in(0, \delta)$,

$$
\mathbb{P}\left[W^{\Gamma}(t)>a\right]<\frac{\epsilon}{2} \int_{0}^{\lambda_{0}} g(\lambda \mid \alpha, p) d \lambda+\int_{\lambda_{0}}^{\infty} g(\lambda \mid \alpha, p) d \lambda \leqslant \epsilon / 2+\epsilon / 2=\epsilon,
$$

which is a contradiction. Hence, $\left\{W^{\Gamma}(t)\right\}_{t \geqslant 0}$ is stochastically continuous. Also, by similar conditioning arguments, it follows that $\left\{\widetilde{W}_{\beta}^{\Gamma}(t)\right\}_{t \geqslant 0}$ is also stochastically continuous.

REMARK 5.2. The Pólya process $\left\{W^{\Gamma}(t)\right\}_{t \geqslant 0}$ and the SFPP $\left\{\widetilde{W}_{\beta}^{\Gamma}(t)\right\}_{t \geqslant 0}$ are not Lévy processes, since they do not have independent increments. To see this, let $0 \leqslant t_{1}<t_{2}<t_{3}<\infty$ and $B_{1}, B_{2}$ be Borel sets. Then

$$
\begin{aligned}
& \mathbb{P}\left[W^{\Gamma}\left(t_{2}\right)-W^{\Gamma}\left(t_{1}\right) \in B_{1} ; W^{\Gamma}\left(t_{3}\right)-W^{\Gamma}\left(t_{2}\right) \in B_{2}\right] \\
& =\mathbb{E}\left[\mathbb{P}\left[N\left(t_{2}, \Gamma\right)-N\left(t_{1}, \Gamma\right) \in B_{1} ; N\left(t_{3}, \Gamma\right)-N\left(t_{2}, \Gamma\right) \in B_{2} \mid \Gamma\right]\right] \\
& =\mathbb{E}\left[\mathbb{P}\left[N\left(t_{2}, \Gamma\right)-N\left(t_{1}, \Gamma\right) \in B_{1} \mid \Gamma\right] \mathbb{P}\left[N\left(t_{3}, \Gamma\right)-N\left(t_{2}, \Gamma\right) \in B_{2} \mid \Gamma\right]\right]
\end{aligned}
$$

and 
(5.8) $\mathbb{P}\left[W^{\Gamma}\left(t_{2}\right)-W^{\Gamma}\left(t_{1}\right) \in B_{1}\right] \mathbb{P}\left[W^{\Gamma}\left(t_{3}\right)-W^{\Gamma}\left(t_{2}\right) \in B_{2}\right]$

$$
=\mathbb{E}\left[\mathbb{P}\left[N\left(t_{2}, \Gamma\right)-N\left(t_{1}, \Gamma\right) \in B_{1} \mid \Gamma\right]\right] \mathbb{E}\left[\mathbb{P}\left[N\left(t_{3}, \Gamma\right)-N\left(t_{2}, \Gamma\right) \in B_{2} \mid \Gamma\right]\right] .
$$

We next show that the right-hand side of (5.7) is not equal to the right-hand side of (5.8). Take, for example, $t_{1}=1, t_{2}=2, t_{3}=3, B_{1}=\{n\}$, and $B_{2}=\{m\}$. Then the right-hand side of (5.7) is

$$
\begin{aligned}
\mathbb{E}[\mathbb{P}[N(1, \Gamma)=n \mid \Gamma] \mathbb{P}[N(1, \Gamma)=m \mid \Gamma]] & =\mathbb{E}\left[\frac{\Gamma^{n} e^{-\Gamma}}{n !} \frac{\Gamma^{m} e^{-\Gamma}}{m !}\right] \\
=\frac{1}{n ! m !} \int_{0}^{\infty} y^{n+m} e^{-2 y} g(y \mid \alpha, p) d y & =\frac{1}{n ! m !} \frac{\alpha^{p}}{\Gamma(p)} \frac{\Gamma(n+m+p)}{(\alpha+2)^{n+m+p}} .
\end{aligned}
$$

Again, the right-hand side of (5.8) takes the form

$$
\begin{aligned}
\mathbb{E}[\mathbb{P}[N(1, \Gamma)=n \mid \Gamma]] \mathbb{E}[\mathbb{P}[N(2, \Gamma) & =m \mid \Gamma]]=\mathbb{E}\left[\frac{\Gamma^{n} e^{-\Gamma}}{n !}\right] \mathbb{E}\left[\frac{\Gamma^{m} e^{-\Gamma}}{m !}\right] \\
& =\frac{1}{n ! m !} \frac{\alpha^{2 p}}{\Gamma^{2}(p)} \frac{\Gamma(n+p) \Gamma(m+p)}{(\alpha+1)^{n+m+2 p}}
\end{aligned}
$$

It can be seen that the last expressions on the right-hand side of (5.9) and (5.101) are different. In a similar way, we can also prove that $\left\{\widetilde{W}_{\beta}^{\Gamma}(t)\right\}_{t \geqslant 0}$ does not have independent increments.

REMARK 5.3. The mean $\mathbb{E}\left[\widetilde{W}_{\beta}^{\Gamma}(t)\right]$ is infinite, which can be seen as follows. The pgf of $\widetilde{W}_{\beta}^{\Gamma}(t)$ is, for $|u| \leqslant 1$,

$$
\mathbb{E}\left[u^{\widetilde{W}_{\beta}^{\Gamma}(t)}\right]=\int_{0}^{\infty} \mathbb{E}\left[u^{\tilde{N}_{\beta}(t, \lambda)}\right] g(\lambda \mid \alpha, p) d \lambda=\int_{0}^{\infty} e^{\lambda^{\beta} t(1-u)^{\beta}} g(\lambda \mid \alpha, p) d \lambda
$$

(see, for example, [22], eq. (2.12)). Now differentiate both sides with respect to $u$, and let $u \rightarrow 1$ to obtain infinity.

5.3. Connections to PDEs. We here discuss some PDE connections associated with the distributions of the SFPP.

First, we establish a result for the process $\left\{\widetilde{W}_{\beta}^{\Gamma}(t)\right\}_{t \geqslant 0}$, similar to (5.4).

THEOREM 5.3. Let $k \in \mathbb{Z}_{+} \backslash\{0\}$. The pmf (5.5) satisfies the following PDE in time variable $t$ :

$$
\frac{\partial^{k}}{\partial t^{k}} \widetilde{\eta}_{\beta}(n \mid t, \alpha, p)=\left(-\frac{\left(1-B_{n}\right)^{\beta} \Gamma(p+\beta)}{\alpha^{\beta} \Gamma(p)}\right)^{k} \widetilde{\eta}_{\beta}(n \mid t, \alpha, p+k \beta)
$$

with $\widetilde{\eta}_{\beta}(n \mid 0, \alpha, p)=1$ if $n=0$ and zero otherwise. 
P r o o f. Note that, from (5.6), we get

$$
\begin{aligned}
\frac{\partial}{\partial t} \widetilde{\eta}_{\beta}(n \mid t, \alpha, p) & =\int_{0}^{\infty} \frac{\partial}{\partial t} \widetilde{p}_{\beta}(n \mid t, y) g(y \mid \alpha, p) d y \\
& =\int_{0}^{\infty}-y^{\beta}\left(1-B_{n}\right)^{\beta} \widetilde{p}_{\beta}(n \mid t, y) g(y \mid \alpha, p) d y \quad \text { (using (5.4)) } \\
& =-\left(1-B_{n}\right)^{\beta} \int_{0}^{\infty} y^{\beta} \widetilde{p}_{\beta}(n \mid t, y) g(y \mid \alpha, p) d y \\
& =-\frac{\left(1-B_{n}\right)^{\beta} \Gamma(p+\beta)}{\alpha^{\beta} \Gamma(p)} \widetilde{\eta}_{\beta}(n \mid t, \alpha, p+\beta),
\end{aligned}
$$

where the last step is due to the fact that

$$
y^{\beta} g(y \mid \alpha, p)=\frac{\Gamma(p+\beta)}{\alpha^{\beta} \Gamma(p)} g(y \mid \alpha, p+\beta) .
$$

Now, repeating the above computation $k$ times, we get the desired result.

COROLlaRY 5.1. The pgf $G_{\beta}(u \mid t, \alpha, p)=\mathbb{E}\left[u^{\widetilde{W}_{\beta}^{\Gamma}(t)}\right],|u| \leqslant 1$, satisfies the following $k$-th order PDE:

$$
\frac{\partial^{k}}{\partial t^{k}} G_{\beta}(u \mid t, \alpha, p)=\left(-(1-u)^{\beta} \frac{\Gamma(p+\beta)}{\alpha^{\beta} \Gamma(p)}\right)^{k} G_{\beta}(u \mid t, \alpha, p+k \beta),
$$

where $G_{\beta}(u \mid 0, \alpha, p)=1$, and $k \in \mathbb{Z}_{+} \backslash\{0\}$.

Proof. Note that

$$
\left(1-B_{n}\right)^{\beta}=\sum_{r=0}^{\infty} \frac{\Gamma(\beta+1)}{\Gamma(r+1) \Gamma(\beta-r+1)}(-1)^{r} B_{n}^{r} .
$$

From (5.11), we obtain

$$
\begin{aligned}
& \frac{\partial}{\partial t} G_{\beta}(u \mid t, \alpha, p) \\
= & \frac{\partial}{\partial t} \sum_{n=0}^{\infty} u^{n} \widetilde{\eta}_{\beta}(n \mid t, \alpha, p)=\sum_{n=0}^{\infty} u^{n}\left(-\left(1-B_{n}\right)^{\beta} \frac{\Gamma(p+\beta)}{\alpha^{\beta} \Gamma(p)}\right) \widetilde{\eta}_{\beta}(n \mid t, \alpha, p+\beta) \\
= & -\frac{\Gamma(p+\beta)}{\alpha^{\beta} \Gamma(p)} \sum_{n=0}^{\infty} u^{n}\left(1-B_{n}\right)^{\beta} \widetilde{\eta}_{\beta}(n \mid t, \alpha, p+\beta) \\
= & -\frac{\Gamma(p+\beta)}{\alpha^{\beta} \Gamma(p)} \sum_{n=0}^{\infty} u^{n} \sum_{r=0}^{\infty} \frac{\Gamma(\beta+1)}{\Gamma(r+1) \Gamma(\beta-r+1)}(-1)^{r} B_{n}^{r} \widetilde{\eta}_{\beta}(n \mid t, \alpha, p+\beta)
\end{aligned}
$$




$$
\begin{aligned}
& =-\frac{\Gamma(p+\beta)}{\alpha^{\beta} \Gamma(p)} \sum_{n=0}^{\infty} u^{n} \sum_{r=0}^{n} \frac{\Gamma(\beta+1)}{\Gamma(r+1) \Gamma(\beta-r+1)}(-1)^{r} \widetilde{\eta}_{\beta}(n-r \mid t, \alpha, p+\beta) \\
& =-\frac{\Gamma(p+\beta)}{\alpha^{\beta} \Gamma(p)} \sum_{r=0}^{\infty} \frac{\Gamma(\beta+1)}{\Gamma(r+1) \Gamma(\beta-r+1)}(-1)^{r} \sum_{n=r}^{\infty} u^{n} \widetilde{\eta}_{\beta}(n-r \mid t, \alpha, p+\beta) \\
& =-\frac{\Gamma(p+\beta)}{\alpha^{\beta} \Gamma(p)} \sum_{r=0}^{\infty} \frac{\Gamma(\beta+1)}{\Gamma(r+1) \Gamma(\beta-r+1)}(-1)^{r} \sum_{n=0}^{\infty} u^{n+r} \widetilde{\eta}_{\beta}(n \mid t, \alpha, p+\beta) \\
& =-(1-u)^{\beta} \frac{\Gamma(p+\beta)}{\alpha^{\beta} \Gamma(p)} G_{\beta}(u \mid t, \alpha, p+\beta) .
\end{aligned}
$$

Taking the derivative $k$ times, we get the result.

Finally, we obtain the following result for the variable $p$.

THEOREM 5.4. The pmf of the SFPP, given in (5.5)), satisfies the following fractional PDE:

$$
\begin{aligned}
& \text { (5.14) } \partial_{p}^{\nu} \widetilde{\eta}_{\beta}(n \mid t, \alpha, p) \\
& =\partial_{p}^{\nu-1}(\log (\alpha)-\psi(p)) \widetilde{\eta}_{\beta}(n \mid t, \alpha, p)+\int_{0}^{\infty} \widetilde{p}_{\beta}(n \mid t, \lambda) \log (\lambda) \partial_{p}^{\nu-1} g(\lambda \mid \alpha, p) d \lambda
\end{aligned}
$$

where $\widetilde{\eta}_{\beta}(n \mid 0, \alpha, p)=1$ if $n=0$ and zero otherwise.

Proof. Note that

$$
\partial_{p}^{\nu} \widetilde{\eta}_{\beta}(n \mid t, \alpha, p)=\partial_{p}^{\nu} \int_{0}^{\infty} \widetilde{p}_{\beta}(n \mid t, \lambda) g(\lambda \mid \alpha, p) d \lambda=\int_{0}^{\infty} \widetilde{p}_{\beta}(n \mid t, \lambda) \partial_{p}^{\nu} g(\lambda \mid \alpha, p) d \lambda .
$$

The theorem now follows from Lemma 4.2.

Acknowledgments. The authors are deeply grateful to the referees for their detailed report and numerous critical comments and suggestions which improved the paper significantly, both in the content and the quality of the paper.

\section{REFERENCES}

[1] G. E. Andrews, R. Askey, and R. Roy, Special Functions, Cambridge University Press, Cambridge 1999.

[2] D. Applebaum, Lévy Processes and Stochastic Calculus, second edition, Cambridge University Press, Cambridge 2009.

[3] L. Beghin, Fractional gamma processes and fractional gamma-subordinated processes, Stoch. Anal. Appl. 33 (2015), pp. 903-926.

[4] L. Beghin and C. Macci, Fractional discrete processes: Compound and mixed Poisson representations, J. Appl. Probab. 51(1) (2014), pp. 9-36.

[5] L. Beghin and E. Orsingher, Fractional Poisson processes and related planar random motions, Electron. J. Probab. 14 (2009), pp. 1790-1827. 
[6] R. Biard and B. Saussereau, Fractional Poisson process: Long-range dependence, applications in ruin theory, J. Appl. Probab. 51 (3) (2014), pp. 727-740.

[7] N. H. Bingham, Limit theorems for occupation times of Markov processes, Z. Wahrsch. Verw. Gebiete 17 (1971), pp. 1-22.

[8] A. Erdélyi, W. Magnus, F. Oberhettinger, and F. G. Tricomi, Higher Transcendental Functions, Vol. 3, McGraw-Hill Book Company Inc., New York-Toronto-London 1955.

[9] W. Feller, An Introduction to Probability Theory and Its Applications, Vol. 2, second edition, Wiley, New York 1971.

[10] R. Gorenflo and F. Mainardi, On the fractional Poisson process and the discretized stable subordinator, Axioms 4 (2015), pp. 321-344.

[11] A. A. Kilbas, M. Saigo, and J. J. Trujillo, On the generalized Wright function, Fract. Calc. Appl. Anal. 5 (2002), pp. 437-460.

[12] A. A. Kilbas, H. M. Srivastava, and J. J. Trujillo, Theory and Applications of Fractional Differential Equations, Elsevier Science, Amsterdam 2006.

[13] T. J. Kozubowski and K. Podgórski, Distributional properties of the negative binomial Lévy process, Probab. Math. Statist. 29 (2009), pp. 43-71.

[14] N. Laskin, Fractional Poisson process, Commun. Nonlinear Sci. Numer. Simul. 8 (2003), pp. 201-213.

[15] N. Laskin, Some applications of the fractional Poisson probability distribution, J. Math. Phys. 50 (2009), 113513.

[16] N. N. Leonenko, M. M. Meerschaert, R. L. Schilling, and A. Sikorskii, Correlation structure of time-changed Lévy processes, Commun. Appl. Ind. Math. 6 (1) (2014), e-483.

[17] F. Mainardi, Fractional Calculus and Waves in Linear Viscoelasticity: An Introduction to Mathematical Models, Imperial College Press, London 2010.

[18] F. Mainardi, R. Gorenflo, and E. Scalas, A fractional generalization of the Poisson processes, Vietnam J. Math. 32 (2004), pp. 53-64.

[19] A. M. Mathai, R. K. Saxena, and H. J. Haubold, The H-Function: Theory and Applications, Springer, New York 2010.

[20] M. M. Meerschaert, E. Nane, and P. Vellais amy, The fractional Poisson process and the inverse stable subordinator, Electron. J. Probab. 16 (2009), pp. 1600-1620.

[21] M. M. Meerschaert and P. Straka, Inverse stable subordinators, Math. Model. Nat. Phenom. 8 (2013), pp. 1-16.

[22] E. Orsingher and F. Polito, The space-fractional Poisson process, Statist. Probab. Lett. 82 (2012), pp. 852-858.

[23] K. Sato, Lévy Processes and Infinitely Divisible Distributions, Cambridge University Press, Cambridge 1999.

[24] N. M. Temme, Special Functions: An Introduction to the Classical Functions of Mathematical Physics, Wiley, New York 1996.

[25] P. Vellais amy and A. Kumar, First-exit times of an inverse Gaussian process, Stochastics 90 (2018), pp. 29-48.

[26] P. Vellaisamy and M. Sreehari, Some intrinsic properties of the gamma distribution, J. Japan Statist. Soc. 40 (2010), pp. 133-144.

Palaniappan Vellaisamy Indian Institute of Technology Bombay

Department of Mathematics

Powai, Mumbai 400076, India

E-mail:pv@math.iitb.ac.in
Aditya Maheshwari Indian Institute of Management Indore Operations Management and Quantitative Techniques Area Indore-453556, Madhya Pradesh, India E-mail: adityam@iimidr.ac.in

Received on 31.10.2013; revised version on 27.10.2016 